\title{
Modelling tumour-immune dynamics, disease progression and treatment
}

Pre-clinical and clinical research indicates that the immune system plays a key role against the development and progression of tumours. One cutting-edge treatment strategy that has rapidly expanded over the past decade is tumour immunotherapy. This involves harnessing the potential of an anti-tumour-immune response to drive sustained cancer remission and ideally a reliable cure.

While incredible efforts have been made over the past decades to decipher the complexity of tumour-immune interactions, there is still a growing need for innovative quantitative modelling approaches that account for the complexity of tumour-immune dynamics. Mathematical modelling can shed light on the multifaceted processes implicated in this new type of therapy, such as the dynamics of immune activation and regulation, immune responses against tumour, tumour suppression of immune cells, the impact of the tumour environment, tumour escape mechanisms and recent advances in cancer therapies.

This special issue of Letters in Biomathematics presents a selection of research papers that mathematically model tumour-immune dynamics, tumour progression and immunotherapeutic treatments using different mathematical modelling approaches ranging from ordinary and partial differential equations, to graph theory and kinetic theory for active particle approach. A total of 8 peer-reviewed original research papers were contributed by a total of 31 co-authors from various academic institutions spanning the US, UK, Italy, Australia and North Africa. They cover a wide spectrum of topics in the tumour-immune dynamics as described below.

The contributions in this special issue show the significance and rapid development of the tumour-immune dynamics research in recent years. With diverse mathematical and biological results, we hope that the readers of the Letters in Biomathematics will find helpful and inspirational directions for their own research in this timely and fast developing area of the tumour-immune dynamics.

Macrophages are one of the most abundant types of immune cells in many solid tumours. Their plasticity, their ability to change phenotype from an anti-tumour M1 phenotype to a pro-tumour M2 phenotype, impacts tumour evolution and many anti-tumour therapies, such as oncolytic virotherapies (OVs). OVs use anti-cancer viruses to eliminate cancer cells. Macrophages are considered to be one of the first lines of defence against viral infections. Their effects on different oncolytic viruses are complex and still poorly understood. In particular, many experimental studies in the literature do not distinguish between various types of macrophages inside the tumour environment, and therefore do not clarify the biological mechanisms of interactions between these different types of macrophages, oncolytic viruses and tumour elimination/growth. Moreover, in the immunological literature there is a debate 
as to whether the overall number or the type of macrophages inside the tumour microenvironment can be used as a prognostic factor for tumour evolution and patient outcome. In 'Tumour-associated macrophages and oncolytic virotherapies: a mathematical investigation into a complex dynamics' by R. Eftimie and G. Eftimie, the authors focus on oncolytic virotherapies to investigate the complex effects of macrophages on different oncolytic viruses. To this end, they develop an ODE-based mathematical approach to obtain a better understanding at a cell population level on the general mechanisms that could explain tumour elimination/control in the presence of oncolytic viruses and an M1-dominated immune response, or an M2-dominated immune response. They show that oncolytic virus therapy can eliminate tumour cells through either (i) anti-tumour-immune activation (in the presence of an M1dominated immune response), or (ii) enhanced oncolysis (in the presence of an M2-dominated immune response). In addition, they demonstrate that tumour reduction and elimination did not depend only on the ratio of M1:M2 cells, but also on the number of tumour-infiltrating macrophages. Overall, in this theoretical study, the authors describe how the various immune scenarios as proposed by different experimental studies, regarding the roles of oncolytic viruses on tumour growth/decay, as well as the roles of number/type of macrophages inside the tumour microenvironment are all possible inside the tumour microenvironment. Moreover, these various scenarios can all be explained with one model as the different tumour-immune-virus interaction rates as well as the rates of M1:M2 re-polarization in macrophages phenotypes are varied. This result emphasizes the power of mathematical modelling for summarizing the possible outcomes of complex biological interactions inside the tumour microenvironment, and for identifying the biological conditions for certain outcomes to take place.

When we catch a virus, such as the flu, our body has an extensive network of cells in place that work to remove the invader. These cells are a subset of what is commonly known as our immune system. It is only over the last few decades that researchers have discovered that our immune system, if stimulated correctly, can be used to kill cancer cells in the body. The tricky part of this cancer treatment, is determining the correct way to stimulate the immune system. One avenue biologists have been investigating is to use viruses to trigger immune cells and stimulate them to kill cancer cells. In 'Modelling combined virotherapy and immunotherapy: strengthening the anti-tumour-immune response mediated by IL-12 and GM-CSF expression' by A.L. Jenner, C.O. Yun, A.Yoon, A.C.F. Coster and P.S. Kim, the authors used an ODE-based mathematical model to optimize current experimental results and to investigate why this therapy works for some cases, but not for others. They discovered that the speed at which the immune cells kills tumour cells is instrumental in determining the outcome of this treatment. Specifically, they note that there is a range of immune cell killing speeds for which the treatment is not effective, but if the immune cells are working outside this range, i.e. very slowly or very quickly, the treatment will be able to eradicate the tumour or at least reduce it to a small size.

Both helper $\mathrm{T}$ and cytotoxic $\mathrm{T}$ cells play a crucial role in the anti-tumour-immune response. How the anti-tumour-immune response varies with the level of infiltrating helper and cytotoxic $\mathrm{T}$ cells is the focus of the paper 'A mathematical model of cytotoxic and helper $\mathrm{T}$ cell interactions in a tumour microenvironment' by H. Dritschel, S.L. Waters, A. Roller and H.M. Byrne. The authors address the heterogeneity of subpopulations of helper and cytotoxic T cells in an anti-tumour immune response by proposing a simple and elegant, yet highly predictive, model consisting of three ordinary differential equations for tumour cells, helper and cytotoxic $\mathrm{T}$ cells. Immunosuppressive effects are implicitly included through a biphasic helper $\mathrm{T}$ cell proliferation term. The model exhibits the three Es of Immunoediting - elimination, equilibrium and escape. A comprehensive analytical and numerical investigation show that infiltration of both helper and cytotoxic T cells control the conditions for tumour elimination. Moreover, the 
results suggest that combined therapies which both block immunosuppressive effects and boost the helper and cytotoxic T cell populations may produce the most favourable outcomes.

The recent advance of single cell RNA sequencing technologies has enabled a new highdimensional definition of cell states. Due to the high-dimensionality of the data (on the order of 20,000), various dimension reduction techniques have been developed to interrogate the data. This new view of biology, presented in 'Modeling acute myeloid leukaemia in a continuum of differentiation states' by H. Cho, K. Ayers, L. de Pillis, Y.-H. Kuo, J. Park, A. Radunskaya and R. Rockne, reveals a continuum, rather than a discrete set, of cell states. In this study, the authors develop a mathematical model of movement in the continuum of states of haematopoietic cell differentiation that is constructed from single cell RNA sequencing data processed with a dimension reduction technique. They represent trajectories in the differentiation space as a graph that represents intermediate states connecting canonical cell states and model directed and random movement on the graph with partial differential equations. The authors use the flow of cell distribution on the graph to describe normal haematopoietic cell differentiation processes as well as predict the evolution of abnormal differentiation processes such as those observed during pathogenesis of acute myeloid leukaemia (AML). While the model incorporates the characteristics of canonical discrete cell state models, it also predicts the dynamics and emergence of novel intermediate cell states.

Viruses are well known for their ability to cause sickness, epidemics and sometimes fatalities; however, there is an upside to these nasty infectious agents. Researchers have discovered that viruses can be re-programmed to attack and kill tumour cells while leaving the rest of the human body alone. In 'Treating cancerous cells with viruses: insights from a minimal model for oncolytic virotherapy' by A.L. Jenner, A.C.F. Coster, P.S. Kim and F. Frascoli, the collaborators use a simple ODE-based mathematical model and analyse the stability of equilibria, oscillations and homoclinic orbits to understand why viruses are effective as a cancer treatment. They find that this style of cancer treatment will always be effective when the tumour has stopped growing or if the human body could be stopped from removing the virus. Unfortunately, these are special cases, and when this treatment is considered on a growing tumour in a normal patient, the complete eradication of the tumour is not possible. On the plus side, the authors discover that for rapidly growing tumours, this treatment can reduce the tumour to a more manageable size for an extended period of time before it starts to grow again. The authors conclude that this treatment has the ability to be made much more effective if it could be combined with other cancer treatments, such as chemotherapy of immunotherapy.

The use of immune checkpoint inhibitors is becoming more commonplace in clinical trials across the nation. Two important factors in the tumour-immune response are the checkpoint protein programmed death-1 (PD-1) and its ligand PD-L1. Also, the effects of intermittent and continuous treatments on tumour-immune dynamics is of great importance to both experimental and quantitative researchers alike. In the paper, 'Tumour-immune dynamics with an immune checkpoint inhibitor' by Elpiniki Nikolopoulou, Lauren R. Johnson, Duane Harris, John D. Nagy, Edward C. Stites and Yang Kuang, the authors derive a mathematical model using a system of ordinary differential equations to study the tumour-immune dynamics with and without the use of anti-PD-1. They consider the system without the anti-PD-1 drug to determine the stability of the tumour-free and tumorous equilibria. Through simulations, they find that a normally functioning immune system might control the tumour. Treatment with anti-PD-1 alone, however, may not be sufficient to eradicate tumour cells. Therefore, it might be beneficial to combine single agent treatments with additional therapies to obtain a better anti-tumour response. 
Cancer treatment protocols have begun to rely increasingly on combination therapies to overcome cancer resistance, but the design of successful combined protocols is still an open problem. Important questions include: How can combination therapies best be used to reduce the emergence of cancer resistance? How might intra-tumoral competition modify the effectiveness of anti-cancer treatments? Bearing these questions in mind, the contribution by $\mathrm{E}$. Piretto, M. Delitala and M. Ferraro, in 'How combination therapies shape drug resistance in heterogeneous tumoural populations' presents a mathematical model of cancer-immune competition under therapies. The authors develop a model consisting of a system of differential equations that describes the dynamics of two cancer types competing for resources and with different susceptibilities to the action of immune system cells and therapies. The model is validated with experimental data, and clinical protocols for non-small cell lung cancer are simulated. The authors simulate two kinds of treatments: a therapy reducing the rate of growth of cancer used alone, and growth-slowing therapy in combination with drugs that increase the effectiveness of the immune system. They conclude that the selection of appropriate infusion schedules plays a key role in the success of anti-cancer therapies. The authors also analyse the outcomes of protocols of chemotherapy and immunotherapy (separately and in combination) differing in doses and timing of the treatments. In particular, they highlight how exploiting the competition between cancer populations seems to be an effective recipe for limiting the emergence of resistant populations. Thus, they note that in some cases the combination of low-dose therapies could yield a substantial control of the total tumour population without imposing massive selective pressure that would suppress the sensitive clones, leaving unchecked the clonal types resistant to therapies.

The immune system is capable of inhibiting the growth of very small tumours and eliminating them before they become clinically recognized - this is also known as the tumour immunosurveillance hypothesis. This early stage is significant since the competition between the tumour and immune cells can still lead to a complete elimination of the tumour cells by the activated immune system. To address these early stage interactions between single cells of the tumour-immune system, the co-authors, M. CH-Chaoui, A. Eladdadi and K. Mokni, present a mathematical model using the kinetic theory for active particle approach in their paper titled 'Activation of the immune response by cytokines and its effect on tumour cells: a mathematical model'. The main advantage of the kinetic theory is that it provides a deeper insight into the interactions between the tumour and immune cells at a cellular level at an early stage of tumour development, that is before the tumour becomes a macroscopically observable spatial structure. This model which describes the tumour-immune competition mediated by the cytokines at the cellular level consists of a system of three coupled nonlinear integro-differential equations. This study focuses on determining the conditions under which the immune activation by cytokines leads to a complete elimination of the tumour cells in their early stage of development. In particular, the focus is on (1) the ability of the tumour cells to inhibit the activated immune cells and (2) the role of the cytokines activation of the immune cells and the progressive decay of the cytokine signals activity.

We would like to express our deepest gratitude to all of the authors for their timely contributions. We are also grateful to the referees for their time and valuable feedback. We thank the Editor-in-Chief of Letters in Biomathematics (LiB), Professor Olcay Akman for his invitation and coordination to edit this special issue. We greatly thank Taylor \& Francis Publisher's editorial team for their professional help, patience, guidance and skilful editing of this special issue. Last but not least, we would like to acknowledge and sincerely thank the Intercollegiate Biomathematics Alliance (IBA) for their financial support to waive the publication fees for our $\mathrm{LiB}$ special issue. 
We are very pleased with this collection of high-quality work and we hope that our readers will find as much fulfilment in these readings as we have.

Amina Eladdadi

The College of Saint Rose, Albany, NY, USA @eladdada@strose.edu (i) http://orcid.org/0000-0002-6813-8650

Lisette de Pillis Harvey Mudd College, Claremont, CA, USA Qdepillis@g.hmc.edu

Peter Kim University of Sydney, Sydney, Australia @ peter.kim@sydney.edu.au 